\title{
Bodies of zero resistance and bodies invisible in one direction
}

\author{
Alena Aleksenko $^{1}$ and Alexander Plakhov ${ }^{1,2}$ \\ ${ }^{1}$ Department of Mathematics, Aveiro University, Aveiro 3810, Portugal \\ 2 Aberystwyth University, Aberystwyth SY23 3BZ, UK
}

Received 15 November 2008, in final form 16 March 2009

Published 23 April 2009

Online at stacks.iop.org/Non/22/1247

Recommended by D Dolgopyat

\begin{abstract}
We consider a body in a parallel flow of non-interacting particles. The interaction of particles with the body is perfectly elastic. We introduce the notions of a body of zero resistance, a body that leaves no trace, and an invisible body, and prove that all such bodies do exist.
\end{abstract}

Mathematics Subject Classification: 37D50, 49Q10

(Some figures in this article are in colour only in the electronic version)

\section{Introduction}

We are concerned with the problem of minimal resistance first addressed by Newton in his Principia (1687): find the body of minimal resistance moving through a medium. The medium particles do not mutually interact, and the interaction of particles with the body is perfectly elastic. Prior to collision with the body, the medium particles are at rest.

The problem can be reformulated in mathematical terms as follows. In an appropriate reference system, the body stays at rest and occupies a bounded connected set with piecewise smooth boundary. There is a parallel flow of point particles falling on the body. The particles behave like billiard balls: they elastically reflect when colliding with the body's boundary, and move freely between consecutive reflections. The resistance to be minimized is calculated by summing up over all momenta imparted to the body by particles hitting the body during a unit time interval.

Since Newton's time, the problem has been considered in various classes of admissible bodies. Initially, the people were primarily interested in studying convex and axially symmetric bodies, assuming additionally that they have fixed length and width [1], or fixed arclength with given base [2,3], or fixed volume [4], etc. Since 1993, the focus of interest shifted to bodies that are generally non-convex and/or non-symmetric [5-12]. It was recently found that in 
some natural classes of non-convex and non-symmetric bodies the infimum of resistance is zero $[13,14]$.

In this paper we report that there exist bodies whose resistance is exactly zero.

More precisely, denoting by $v_{0} \in S^{2}$ the initial flow velocity, we say that the body has zero resistance in the direction $v_{0}$ if the final velocity of almost every particle is also equal to $v_{0}$. We say that the body leaves no trace (or is trackless) in the direction $v_{0}$ if it has zero resistance in this direction and, additionally, the flow density behind the body is constant and coincides with the initial one. Further, we say that the body is invisible in the direction $v_{0}$ if the trajectory of each particle outside a prescribed bounded set coincides with a straight line. Indeed, such a body with a mirrored surface becomes invisible to an observer staying in this direction far enough, or if illuminated only by parallel rays in the direction $v_{0}$. We prove that there exist bodies of zero resistance, bodies leaving no trace and bodies invisible in one direction.

From the viewpoint of classical scattering by obstacle, we construct a body with zero total cross section. (The total cross section measures the density of scattered rays; see the introduction to [15] for the definition.) Thus in this case, from the scattering data for a fixed angle of incidence, it is not possible even to say whether an obstacle exists or not. The question arises as to whether a similar effect can take place in the wave scattering or in the nonrelativistic quantum mechanics. We note in this regard that the classical and wave scattering at small wavelength are closely connected (see, e.g. [16]).

The paper is organized as follows. In section 2, we introduce the mathematical notation and give rigorous definitions for bodies of zero resistance, bodies that leave no trace and bodies that are invisible in one direction. In section 3 , we give an overview of the minimal resistance problem and put our result in this context. In section 4 , we introduce families of zero resistance bodies, trackless bodies and invisible bodies, discuss their properties, and state some open problems. Finally, in section 5 possible applications of our models are discussed.

\section{Notation and definitions}

Let $\mathcal{B} \subset \mathbb{R}^{3}$ be a bounded connected set with a piecewise smooth boundary, and let $v_{0} \in S^{2}$. ( $\mathcal{B}$ and $v_{0}$ represent the body and the flow direction, respectively.) Consider the billiard in $\mathbb{R}^{3} \backslash \mathcal{B}$. The scattering mapping $(x, v) \mapsto\left(x_{\mathcal{B}}^{+}(x, v), v_{\mathcal{B}}^{+}(x, v)\right)$ from a full measure subset of $\mathbb{R}^{3} \times S^{2}$ into $\mathbb{R}^{3} \times S^{2}$ is defined as follows. Let the motion of a billiard particle $x(t), v(t)$ satisfy the relations $x(t)=\left\{\begin{array}{ll}x+v t, & \text { if } t<t_{1} \\ x^{+}+v^{+} t, & \text { if } t>t_{2}\end{array}\right.$ and $v(t)=\left\{\begin{array}{ll}v, & \text { if } t<t_{1} \\ v^{+}, & \text {if } t>t_{2}\end{array}\right.$ (here $t_{1}, t_{2}$ are a pair of real numbers depending on the particular motion); then $x^{+}=: x_{\mathcal{B}}^{+}(x, v), v^{+}=: v_{\mathcal{B}}^{+}(x, v)$.

Denote $\tilde{x}_{\mathcal{B}}^{+}(x, v)=x^{+}-\left\langle x^{+}, v^{+}\right\rangle v^{+}$and $t^{*}=t_{\mathcal{B}}^{*}(x, v)=-\left\langle x^{+}, v^{+}\right\rangle$, where $\langle\cdot, \cdot\rangle$ is the scalar product; then one has $x^{+}+v^{+} t=\tilde{x}^{+}+v^{+}\left(t-t^{*}\right)$, and $\tilde{x}^{+}$is orthogonal to $v^{+}$. We also denote by $\{v\}^{\perp}$ the orthogonal complement to the one-dimensional subspace $\{v\}$, that is, the plane that contains the origin and is orthogonal to $v$.

\section{Definition.}

$\mathbf{D}_{1}$. We say that $\mathcal{B}$ has zero resistance in the direction $v_{0}$ if $v_{\mathcal{B}}^{+}\left(x, v_{0}\right)=v_{0}$ for almost every $x$.

$\mathbf{D}_{2}$. We say that the body $\mathcal{B}$ leaves no trace in the direction $v_{0}$ if, additionally to $\mathrm{D}_{1}$, the mapping $x \mapsto \tilde{x}_{\mathcal{B}}^{+}\left(x, v_{0}\right)$ from a subset of $\left\{v_{0}\right\}^{\perp}$ into $\left\{v_{0}\right\}^{\perp}$ is defined almost everywhere in $\left\{v_{0}\right\}^{\perp}$ and preserves the two-dimensional Lebesgue measure. 
$\mathbf{D}_{3}$. We say that $\mathcal{B}$ is invisible in the direction $v_{0}$ if, additionally to $\mathrm{D}_{2}$, one has $\tilde{x}_{\mathcal{B}}^{+}\left(x, v_{0}\right)=x$.

The condition $D_{3}$ is stronger than $D_{2}$, and $D_{2}$ is stronger than $D_{1}$. One easily sees that if $\mathcal{B}$ is invisible/leaves no trace in the direction $v_{0}$ then the same is true in the opposite direction $-v_{0}$.

This definition is interpreted as follows. Suppose that there is a parallel flow of noninteracting particles falling on $\mathcal{B}$. Initially, the velocity of a particle equals $-v_{0}$; then it makes several reflections from $\mathcal{B}$, and finally moves freely with the velocity $v_{\mathcal{B}}^{+}\left(x, v_{0}\right)$, where $x$ indicates the initial position of the particle. One can imagine that the flow is highly rarefied or consists of rays of light. (Equivalently, one can assume that the body translates at the velocity $-v_{0}$ through a highly rarefied medium of particles at rest.) The force of pressure of the flow on the body (or the force of resistance of the medium to the body's motion) is proportional to $R_{v_{0}}(\mathcal{B}):=\int_{\left\{v_{0}\right\}^{\perp}}\left(v_{0}-v_{\mathcal{B}}^{+}\left(x, v_{0}\right)\right) \mathrm{d} x$, where the ratio equals the density of the flow/medium and $\mathrm{d} x$ means the Lebesgue measure in $\left\{v_{0}\right\}^{\perp}$.

In the case $\mathrm{D}_{1}$ one has $R_{v_{0}}(\mathcal{B})=0$. If the body has mirror surface then in the case $\mathrm{D}_{3}$ it is invisible in the direction $v_{0}$. In the case $\mathrm{D}_{2}$, if the body moves through a rarefied medium, the medium seems to be unchanged after the body has passed: the particles behind the body (actually, in the complement of the body's convex hull) are at rest and are distributed with the same density.

In section 4 we give examples of a body satisfying the condition $D_{1}$, but not satisfying $D_{2}$; a body satisfying $\mathrm{D}_{2}$ but not $\mathrm{D}_{3}$; and a body satisfying $\mathrm{D}_{3}$. That is, there exists a body of zero resistance that leaves a trace (shown on figure $2(a)$ ); a body leaving no trace but not invisible (figures 2(b) and (c)); and an invisible body (figure 3).

\section{Problems of the body of minimal resistance}

The origin of least resistance problems goes back to the book Principia (1687) by Newton. Here we give an (incomplete) overview of these problems.

Consider a three-dimensional space $\mathbb{R}_{x_{1}, x_{2}, x_{3}}^{3}$ with orthogonal coordinates $x_{1}, x_{2}, x_{3}$, a bounded two-dimensional set $\Omega \subset \mathbb{R}_{x_{1}, x_{2}}^{2}$ with piecewise smooth boundary and a positive number $h$.

(1) Let $\Omega$ be the unit circle $x_{1}^{2}+x_{2}^{2} \leqslant 1$. Consider the class of bodies $\mathcal{B}$ that are (i) bounded from above by a function $f: \Omega \rightarrow[0, h]$ and (ii) such that any billiard particle in $\mathbb{R}^{3} \backslash \mathcal{B}$ with the initial velocity $v_{0}=(0,0,-1)$ makes at most one reflection from $\partial \mathcal{B}$. Condition (i) implies that $\mathcal{B}$ contains the graph $\left\{\left(x_{1}, x_{2}, x_{3}\right):\left(x_{1}, x_{2}\right) \in \Omega, x_{3}=f\left(x_{1}, x_{2}\right)\right\}$ and is contained in the subgraph $\left\{\left(x_{1}, x_{2}, x_{3}\right):\left(x_{1}, x_{2}\right) \in \Omega, x_{3} \leqslant f\left(x_{1}, x_{2}\right)\right\}$. Condition (ii) is called a single impact assumption; under this assumption, $R_{v_{0}}$ allows a comfortable analytical functional representation: $R_{v_{0}}(\mathcal{B})=\iint_{\Omega}\left(1+|\nabla f|^{2}\right)^{-1} \mathrm{~d} x_{1} \mathrm{~d} x_{2}$. Without loss of generality one can assume that the body is given by the relation $0 \leqslant x_{3} \leqslant f\left(x_{1}, x_{2}\right)$.

There have been studied the minimization problem for the absolute value of the third component of the vector $R_{v_{0}}(\mathcal{B})=\left(R_{v_{0}}^{(1)}, R_{v_{0}}^{(2)}, R_{v_{0}}^{(3)}\right)$ in several subclasses of this class of bodies. These subclasses, (1a)-(1d), are defined by additional conditions on $f$.

(1a) $f$ is concave and radial. This problem was considered by Newton in [1]; the optimal body is indicated there without a proof.

(1b) $f$ is concave. Thus, the class of bodies is larger than in the case 1a. The corresponding minimization problem has been studied since 1993 (see, e.g. [5-9]) and is not completely solved until now. The solution is known to exist and not coincide with the Newtonian one; at any points of its surface, the gaussian curvature either equals zero or does not exist. The solution has been obtained numerically in [9]. 
(1c) $f$ is concave and developable [10]. More precisely, the level set $\{f=h\}$ is nonempty, and the smallest concave function $\tilde{f}$ such that $\{\tilde{f}=h\}=\{f=h\}$ coincides with $f$. The corresponding solution is given in [10].

(1d) $f$ is arbitrary (still under the single impact assumption); this problem has been considered in $[11,12]$.

In all these cases, the infimum of resistance is positive, $\inf _{\mathcal{B}}\left|R_{v_{0}}^{(3)}(\mathcal{B})\right|>0$.

(2) Consider the class of bodies $\mathcal{B}$ that are contained in the cylinder $\Omega \times[0, h]$ and contain a cross section $\Omega \times\{c\}$ with $c \in[0, h], \Omega \times\{c\} \subset \mathcal{B} \subset \Omega \times[0, h]$, and such that the integral $R_{v_{0}}(\mathcal{B})$ exists. For the sake of brevity, we shall call them bodies inscribed in the cylinder. Multiple reflections are allowed. If $\Omega$ is the unit circle then the infimum of resistance equals zero, $\inf _{\mathcal{B}}\left|R_{v_{0}}(\mathcal{B})\right|=0$ (see [14]). This result generalizes to the case of arbitrary $\Omega$, so that the following statement holds true.

Conjecture. For any $\Omega$ and $h, \inf \left\{\left|R_{v_{0}}(\mathcal{B})\right|: \mathcal{B}\right.$ is inscribed in the cylinder $\left.\Omega \times[0, h]\right\}=$ 0 . If $\Omega$ is convex then the infimum is not attained. On the other hand, for some non-convex $\Omega$ and some $h$, the infimum is attained; that is, there exist bodies of zero resistance.

We call it conjecture, since the proof of the first assertion has never been published. The second assertion in the conjecture is reformulated and proved as proposition 1. Several examples of zero resistance bodies, where $\Omega$ is a ring or a special kind of polygon with mutually orthogonal sides, are provided below in the text. This proves the third assertion.

Proposition 1. Let $\Omega$ be a convex set with non-empty interior and let $\mathcal{B}$ be a body inscribed in the cylinder $\Omega \times[0, h]$ and such that the integral $R_{v_{0}}(\mathcal{B})$ exists. Then $R_{v_{0}}(\mathcal{B}) \neq 0$.

Proof. The integral $R_{v_{0}}(\mathcal{B})$ exists, that is, the function $v_{\mathcal{B}}^{+}\left(x, v_{0}\right)$ is defined for almost all $x \in \Omega$ and is measurable. Using that the particle trajectory does not intersect the section $\Omega \times\{c\}$ and $\Omega$ is convex, one concludes that the particle initially moves in the cylinder above this section, then intersects the lateral surface of the cylinder and moves freely afterwards. This implies that $v_{\mathcal{B}}^{+}\left(x, v_{0}\right) \neq v_{0}$, hence $R_{v_{0}}(\mathcal{B}) \neq 0$.

\section{Zero resistance bodies and invisible bodies}

The main result of this paper is the following theorem. Fix $v_{0} \in S^{2}$.

Theorem 1. There exist (a) a body that has zero resistance in the direction $v_{0}$ but leaves a trace; (b) a body that leaves no trace in the direction $v_{0}$ but is not invisible; (c) a body invisible in the direction $v_{0}$.

\section{Proof.}

(a) Consider two identical coplanar equilateral triangles $\mathrm{ABC}$ and $\mathrm{A}^{\prime} \mathrm{B}^{\prime} \mathrm{C}^{\prime}$, with $\mathrm{C}$ being the midpoint of the segment $\mathrm{A}^{\prime} \mathrm{B}^{\prime}$, and $\mathrm{C}^{\prime}$, the midpoint of $\mathrm{AB}$. The vertical line $\mathrm{CC}^{\prime}$ is parallel to $v_{0}$. Let $\mathrm{A}^{\prime \prime}\left(\mathrm{B}^{\prime \prime}\right)$ be the point of intersection of segments $\mathrm{AC}$ and $\mathrm{A}^{\prime} \mathrm{C}^{\prime}\left(\mathrm{BC}\right.$ and $\mathrm{B}^{\prime} \mathrm{C}^{\prime}$, respectively); see figure 1 . The body $\mathcal{B}$ generated by rotation of the triangle $\mathrm{AA}^{\prime} \mathrm{A}^{\prime \prime}$ (or $\left.\mathrm{BB}^{\prime} \mathrm{B}^{\prime \prime}\right)$ around the axis $\mathrm{CC}^{\prime}$ is shown in figure $2(a)$. It has zero resistance in the direction $v_{0}$. This can be better seen from figure 1 representing a vertical central cross section of $\mathcal{B}$.

If a particle initially belongs to this cross section, it will never leave it. Let the particle first hit the segment $\mathrm{A}^{\prime} \mathrm{A}^{\prime \prime}$ at a point $\mathrm{E}$. (If the particle first hits $\mathrm{B}^{\prime} \mathrm{B}^{\prime \prime}$, the argument is 


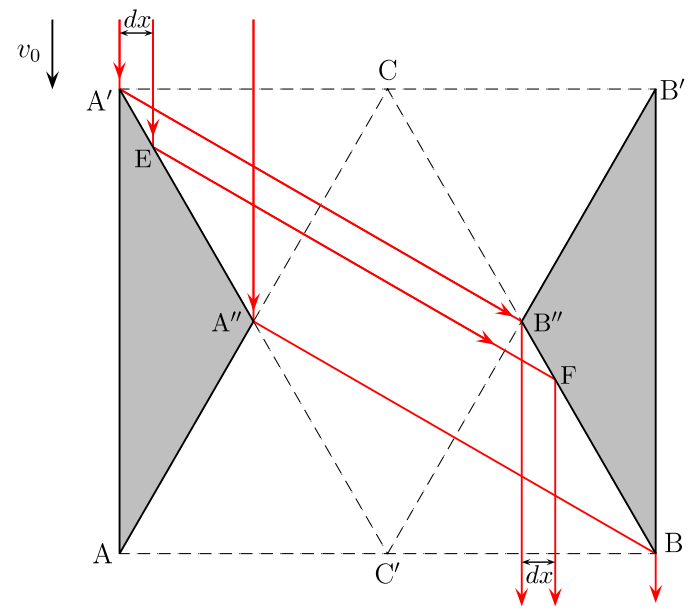

Figure 1. The basic construction

(a)

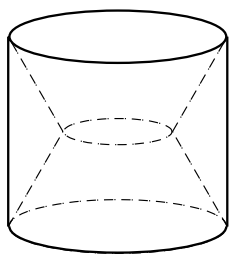

(b)

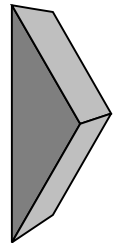

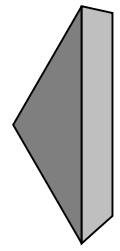

(c)

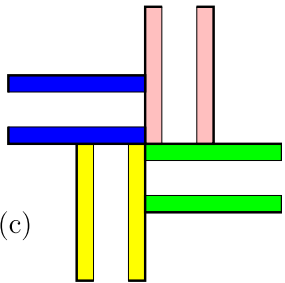

Figure 2. (a) A rotationally symmetric body of zero resistance. (b) A disconnected set leaving no trace. $(c)$ The union of four sets identical to the one shown in figure $(b)$, the above view. It is simply connected and leaves no trace.

the same.) After the reflection, the direction of motion forms the angle $\pi / 3$ with the vertical. Next, the particle hits the segment $B^{\prime \prime} B$ at the point $F$ such that $\left|A^{\prime} E\right|=\left|B^{\prime \prime} F\right|$, and after the second reflection moves vertically downward. That is, the final velocity equals $v_{0}$.

However, this body does leave a trace (and therefore is not invisible). Indeed, the particles that initially belong to a larger cylindrical layer of width $\mathrm{d} x$ (on figure 1 above), after two reflections get into a smaller layer of the same width $\mathrm{d} x$ (figure 1 below), and vice versa. Therefore, the density of the smaller layer gets larger below the body, and the density of the larger layer gets smaller. If $\mathrm{d} x$ is small then the increase and the decrease in the density is twofold.

(b) A set generated by translating the pair of triangles $\mathrm{AA}^{\prime} \mathrm{A}^{\prime \prime}$ and $\mathrm{BB}^{\prime} \mathrm{B}^{\prime \prime}$ along a segment orthogonal to their plane leaves no trace in the vertical direction $v_{0}$, but is not invisible. It is disconnected; however, by 'gluing together' four copies of this set along the vertical faces, one can get a connected set (that is, a true body) leaving no trace. Figure 2(c) provides the above view of the resulting body.

(c) A body invisible in the direction $v_{0}$ can be obtained by doubling a zero resistance body; see figure 3.

Note that the interior of this body is a disjoint union of two domains; this property can be undesirable. However, the construction can be improved as follows. 


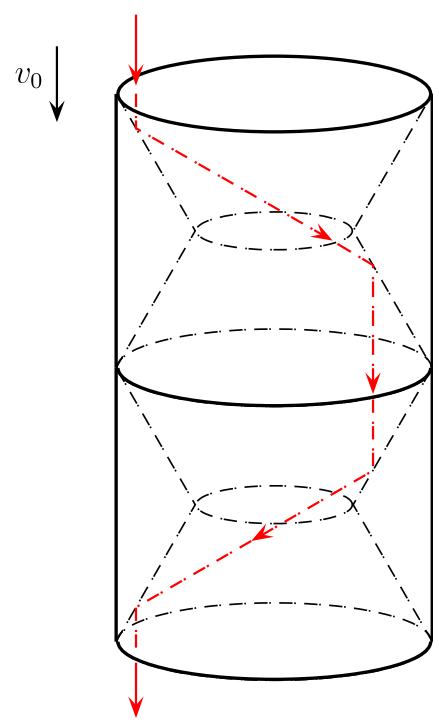

Figure 3. A body invisible in the direction $v_{0}$. It is obtained by taking four truncated cones out of the cylinder.

Consider a coordinate system $O x_{1} x_{2} x_{3}$ such that the $x_{3}$-axis coincides with the symmetry axis of the body $\mathcal{B}$ shown in figure $2(a)$, the upper half-space contains the body and $v_{0}=(0,0,-1)$. Consider the body $\mathcal{B}^{\prime}$ symmetric to $\mathcal{B}$ with respect to the horizontal plane $x_{3}=0$ and suppose that the $\operatorname{distance} \operatorname{dist}\left(\mathcal{B}, \mathcal{B}^{\prime}\right)=: \varepsilon$ is small. Next, take the intersection of $\mathcal{B} \cup \mathcal{B}^{\prime}$ with the set $x_{1} x_{2} \geqslant 0$ (this intersection is the disjoint union of 4 connected sets) and shift it vertically up or down on $2 \varepsilon$. The union of the shifted set with the remaining set $\left(\mathcal{B} \cup \mathcal{B}^{\prime}\right) \cap\left\{x_{1} x_{2} \leqslant 0\right\}$ is connected, that is, it is a true body invisible in the direction $v_{0}$.

Let us introduce some families of bodies having the desired properties. First, consider a pair of isosceles triangles with the angles $\alpha, \alpha$ and $\pi-2 \alpha$, where $0<\alpha<\pi / 4$. The triangles are symmetric to each other with respect to a certain point. This point lies on the symmetry axis of each triangle, at the distance $(\tan 2 \alpha-\tan \alpha) / 2$ from its obtuse angle and at the distance $(\tan 2 \alpha+\tan \alpha) / 2$ from its base. The length of the base of each triangle equals 2 . In figure 4 there are depicted two pairs of triangles, with $\alpha$ small and $\alpha$ close to $\pi / 4$.

As seen from the picture, this definition guarantees zero resistance in the direction $v_{0}$ parallel to the bases of the triangles. The zero resistance body, trackless body and invisible body are created, respectively, by the procedures of rotation, translation with gluing and doubling, applied to the pair of triangles.

Consider the one parameter family of zero resistance bodies $\mathcal{B}_{\alpha}$ obtained by rotation of the pair of triangles. It contains the body $\mathcal{B}=\mathcal{B}_{\pi / 6}$ constructed above. Before studying the properties of this family, introduce the following definition.

For a body $\mathcal{D}$, let $\kappa(\mathcal{D})$ be the relative volume of $\mathcal{D}$ in its convex hull, that is, $\kappa(\mathcal{D}):=$ $\operatorname{Vol}(\mathcal{D}) / \operatorname{Vol}(\operatorname{Conv} \mathcal{D})$. One obviously has $0<\kappa(\mathcal{D}) \leqslant 1$, and $\kappa(\mathcal{D})=1$ iff $\mathcal{D}$ is convex.

The convex hull of $\mathcal{B}_{\alpha}$ is a cylinder of radius $L_{\alpha}=(\tan 2 \alpha+\tan \alpha) / 2$ and height $H=2$; denote by $h_{\alpha}$ its relative height, $h_{\alpha}=H / L_{\alpha}$. One has $\operatorname{Vol}\left(\mathcal{B}_{\alpha}\right)=\pi \tan \alpha(\tan 2 \alpha+\tan \alpha / 3)$. Now one easily derives the asymptotic relations for $h_{\alpha}$ and $\kappa_{\alpha}=\kappa\left(\mathcal{B}_{\alpha}\right)$ : as $\alpha \rightarrow 0$, one has $h_{\alpha}=4 / 3 \alpha(1+o(1)) \rightarrow \infty$ and $\kappa_{\alpha} \rightarrow 14 / 27 \approx 0.52$. For $\alpha=\pi / 6$, one has $h_{\pi / 6}=\sqrt{3}$ and $\kappa_{\pi / 6}=5 / 12 \approx 0.42$. Taking $\alpha=(\pi-\varepsilon) / 4, \varepsilon \rightarrow 0^{+}$, one gets $h_{(\pi-\varepsilon) / 4}=2 \varepsilon(1+o(1))$ and $\kappa_{\alpha}=\varepsilon(1+o(1))$. 


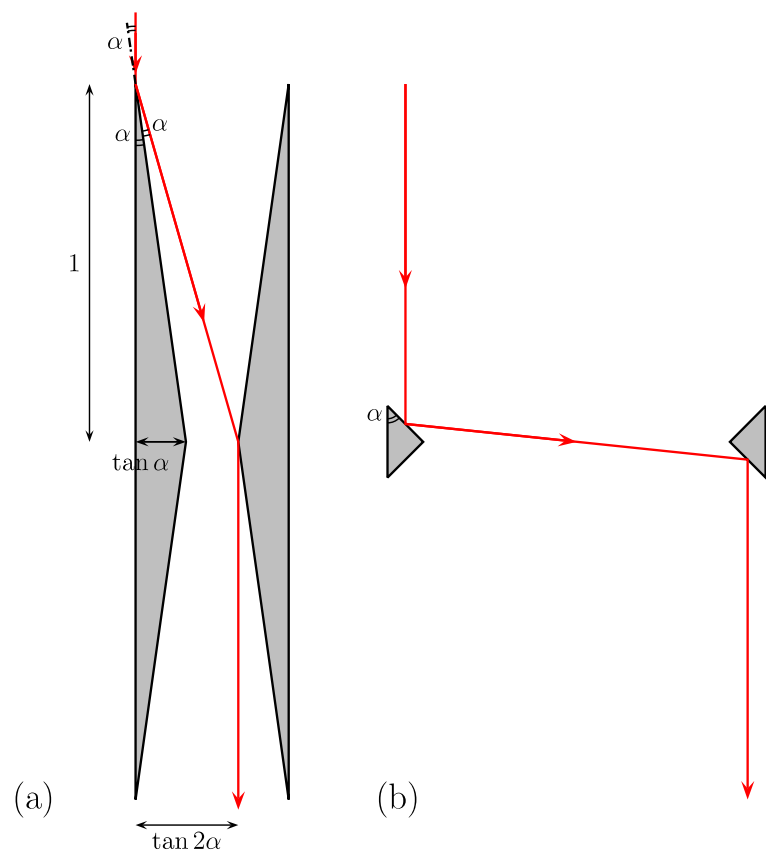

Figure 4. The central vertical cross section of the body $\mathcal{B}_{\alpha}(a)$ with small $\alpha ;(b)$ with $\alpha$ close to $\pi / 4$.

Now consider a more general construction based on the union of two isosceles trapezia $\mathrm{ABCD}$ and $\mathrm{A}^{\prime} \mathrm{B}^{\prime} \mathrm{C}^{\prime} \mathrm{D}^{\prime}$ (see figure 5). Take a billiard particle in $\mathbb{R}^{2} \backslash\left(\mathrm{ABCD} \cup \mathrm{A}^{\prime} \mathrm{B}^{\prime} \mathrm{C}^{\prime} \mathrm{D}^{\prime}\right)$ with the initial velocity directed vertically downwards and having at least one reflection from one of the trapezia. In this and the two next paragraphs we consider the part of the trajectory contained in the trapezia $\mathrm{BB}^{\prime} \mathrm{C}^{\prime} \mathrm{C}$ (see figure $6(a)$ ). The particle gets in through the segment $\mathrm{BB}^{\prime}$, and after a finite number of alternating reflections from the sides $\mathrm{BC}$ and $\mathrm{B}^{\prime} \mathrm{C}^{\prime}$, it escapes through $\mathrm{CC}^{\prime}$ or $\mathrm{BB}^{\prime}$. Suppose without loss of generality that the first reflection takes place from $\mathrm{BC}$, and apply the procedure of unfolding to the billiard trajectory. First, to both the trapezium $\mathrm{BB}^{\prime} \mathrm{C}^{\prime} \mathrm{C}$ and the part of the trajectory after the first reflection, apply the reflection from the line $\mathrm{BC}$. As a result we obtain the trapezium $\mathrm{B}_{1} \mathrm{BCC}_{1}$ and a billiard trajectory in it, where the first segment of the trajectory (between $\mathrm{BC}$ and the next point of reflection) belongs to the same vertical line as the initial part of the particle trajectory.

If the next reflection takes place from the side $\mathrm{B}_{1} \mathrm{C}_{1}$ (as on figure 6(a)) then to both the trapezium $\mathrm{B}_{1} \mathrm{BCC}_{1}$ and the rest of the trajectory (after this reflection and before escaping the trapezium) apply the reflection with respect to the line $\mathrm{B}_{1} \mathrm{C}_{1}$. As a result, we obtain the trapezium $\mathrm{B}_{2} \mathrm{~B}_{1} \mathrm{C}_{1} \mathrm{C}_{2}$ and a billiard trajectory in it, and again, the initial segment of this trajectory belongs to the same vertical line as above.

This procedure ends in a finite number of steps-as a result we obtain a sequence of trapezia $\mathrm{B}_{1} \mathrm{BCC}_{1}, \mathrm{~B}_{2} \mathrm{~B}_{1} \mathrm{C}_{1} \mathrm{C}_{2}, \ldots, \mathrm{B}_{k} \mathrm{~B}_{k-1} \mathrm{C}_{k-1} \mathrm{C}_{k}, k \leqslant\lfloor\pi /(2 \alpha)+1 / 2\rfloor$ and the 'unfolded' part of the trajectory. This unfolded trajectory is a vertical segment whose initial endpoint belongs to $\mathrm{BB}^{\prime}$ and the final endpoint belongs either (i) to the broken line $\mathrm{CC}_{1} \mathrm{C}_{2}, \ldots, \mathrm{C}_{k}$, or (ii) to the broken line $\mathrm{BB}_{1} \mathrm{~B}_{2}, \ldots, \mathrm{B}_{k}$. Case (i) means that the original billiard trajectory intersects $\mathrm{CC}^{\prime}$ and enters the rectangle $\mathrm{CDD}^{\prime} \mathrm{C}^{\prime}$. Case (ii) means that it eventually escapes $\mathrm{BB}^{\prime} \mathrm{C}^{\prime} \mathrm{C}$ through the segment $\mathrm{BB}^{\prime}$. 


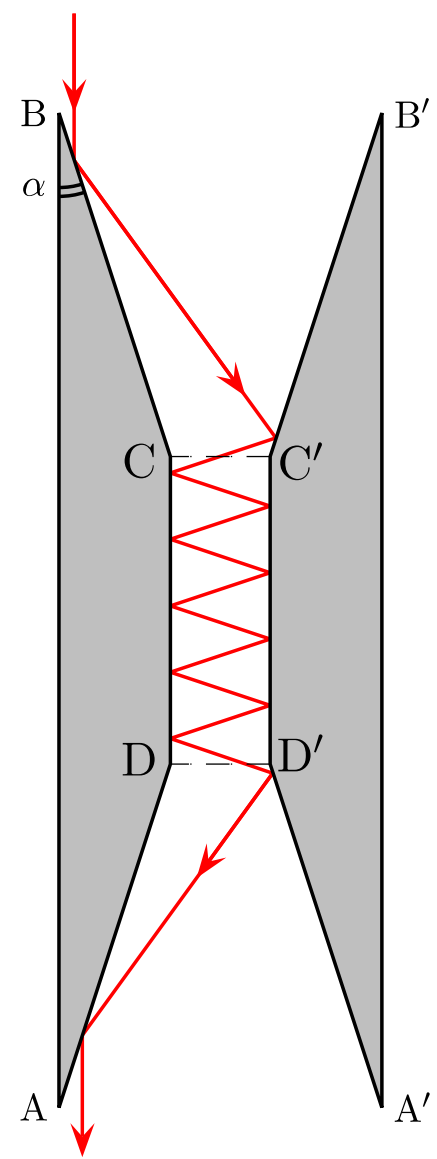

Figure 5. The vertical cross section of a zero resistance body of revolution.

Denote $r:=\left|\mathrm{CC}^{\prime}\right| /\left|\mathrm{BB}^{\prime}\right|$ and $\alpha:=\measuredangle \mathrm{ABC}$ (and therefore $\alpha=\measuredangle \mathrm{BAD}=\measuredangle \mathrm{A}^{\prime} \mathrm{B}^{\prime} \mathrm{C}^{\prime}=$ $\left.\measuredangle \mathrm{B}^{\prime} \mathrm{A}^{\prime} \mathrm{D}^{\prime}\right)$; we assume that $\alpha<\pi / 4$. Choose the parameters $r$ and $\alpha$ in such a way that the broken line $\mathrm{CC}_{1} \mathrm{C}_{2}, \ldots, \mathrm{C}_{\lfloor\pi /(2 \alpha)+1 / 2\rfloor}$ touches the straight line $\mathrm{AB}$, that is, intersects this line and is located to the right of it (see figure 6(a)). It suffices to put $r=r(\alpha)=$ $\sin \alpha / \sin (2\lfloor\pi /(4 \alpha)\rfloor \alpha+\alpha)$. The function $r(\alpha)$ is continuous and monotonically increases from $r(0)=0$ to $r(\pi / 4)=1$. With this choice, case (ii) is excluded, that is, the particle always enters the rectangle $\mathrm{CDD}^{\prime} \mathrm{C}^{\prime}$.

After the first reflection the particle velocity forms the angle $2 \alpha$ with the vertical direction $(0,-1)$ (we measure angles counterclockwise from the vertical); after the second reflection the angle becomes $-4 \alpha$, and so on. At the point of intersection with $\mathrm{CC}^{\prime}$ the angle becomes $(-1)^{k-1} 2 k \alpha$, where $k$ is the number of reflections from $\mathrm{BC}$ and $\mathrm{B}^{\prime} \mathrm{C}^{\prime}$.

When the particle belongs to the rectangle $\mathrm{CC}^{\prime} \mathrm{D}^{\prime} \mathrm{D}$, the modulus of the angle remains equal to $2 k \alpha$, and when the particle makes reflections from the sides $\mathrm{AD}$ and $\mathrm{A}^{\prime} \mathrm{D}^{\prime}$, this value decreases, taking successively the values $2(k-1) \alpha, 2(k-2) \alpha, \ldots$, and finally, after the last reflection, the angle becomes $2 k^{\prime} \alpha$, where $k^{\prime}$ is an integer, $\left|k^{\prime}\right| \leqslant k$.

Let us show that $k^{\prime}=0$ and, therefore, the final velocity is vertical. To that end, let us apply the unfolding procedure again, this time to the part of the trajectory contained in the trapezium $\mathrm{ADD}^{\prime} \mathrm{A}^{\prime}$ (see figure 6(b)). Suppose without loss of generality that the point of last 

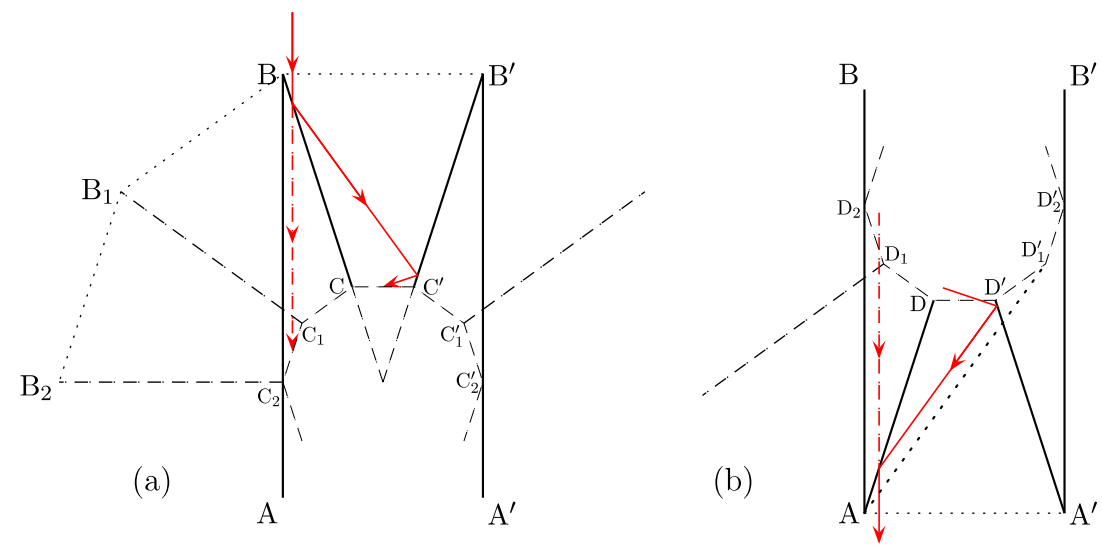

Figure 6. Unfolding of a billiard trajectory.

reflection belongs to $\mathrm{AD}$. To both the part of the trajectory before that point and the trapezium, apply reflection with respect to the line $\mathrm{AD}$. Repeating this procedure as described above, one obtains the 'rectified' trajectory - an interval with the endpoints on the segment $\mathrm{AA}^{\prime}$ ' and on the broken line $\ldots \mathrm{D}_{2} \mathrm{D}_{1} \mathrm{DD}^{\prime} \mathrm{D}_{1}^{\prime} \mathrm{D}_{2}^{\prime} \ldots$ generated by the consecutive reflections of the unfolding procedure. This broken line touches the lines $\mathrm{AB}$ and $\mathrm{A}^{\prime} \mathrm{B}^{\prime}$.

We see that the tangents drawn from $\mathrm{A}$ to the broken line (the lines $\mathrm{AD}_{2}$ and $\mathrm{AD}_{1}^{\prime}$ on figure $6(b)$ ) form the angles 0 and $-2 \alpha$ with the vertical. Analogously, the angles of the tangents drawn from $\mathrm{A}^{\prime}$ to that line are $2 \alpha$ and 0 . This implies that both the tangents drawn from any point of the segment $\mathrm{AA}^{\prime}$ to that line have angles greater than $-2 \alpha$ and less than $2 \alpha$. The same is true for the angle of inclination $2 k^{\prime} \alpha$ of the unfolded trajectory: $-2 \alpha<2 k^{\prime} \alpha<2 \alpha$, and therefore, $k^{\prime}=0$.

The body of zero resistance is formed by rotation of the trapezia around the vertical symmetry axis. Its shape is determined by the two parameters $\alpha$ and $\gamma=|\mathrm{CD}| /|\mathrm{BC}|$. As $\alpha \rightarrow 0$ and $\gamma \rightarrow \infty$, the maximal number of reflections goes to infinity, the relative volume of the body in the cylinder $\mathrm{ABB}^{\prime} \mathrm{A}^{\prime}$ goes to 1 and the relative height of the cylinder goes to infinity.

By doubling this body, one obtains the body invisible in the direction $v_{0}$.

This result can be summarized as follows.

Theorem 2. Let $\Omega$ be a ring $r^{2} \leqslant x_{1}^{2}+x_{2}^{2} \leqslant 1$. For $h$ sufficiently large, there exists a body inscribed in $\Omega \times[0, h]$ and invisible in the direction $v_{0}=(0,0,-1)$.

Remark 1. This theorem is also true for the case where $\Omega$ is a special kind of polygon with mutually orthogonal sides; see, e.g., figure 2(c).

Denote by $m=m\left(\mathcal{B}, v_{0}\right)$ the maximal number of reflections of an individual particle from the body.

Proposition 2. (a) If the body $\mathcal{B}$ has zero resistance or leaves no trace in the direction $v_{0}$ then $m\left(\mathcal{B}, v_{0}\right) \geqslant 2$. (b) If $\mathcal{B}$ is invisible in the direction $v_{0}$ then $m\left(\mathcal{B}, v_{0}\right) \geqslant 4$. These inequalities are sharp: there exist zero resistance bodies and trackless bodies with exactly 2 reflections, and there exist invisible bodies with exactly 4 reflections. 


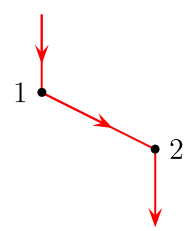

Figure 7. Two reflections are not enough for an invisible body.

\section{Proof.}

(a) If $m=1$ (that is, under the single impact assumption) then the final velocity of each particle does not coincide with the initial one, $v_{\mathcal{B}}^{+}\left(x, v_{0}\right) \neq v_{0}$, therefore $R_{v_{0}}(\mathcal{B}) \neq 0$. That is, a zero resistance body requires at least two reflections.

(b) Note that a thin parallel beam of particles changes the orientation under each reflection. To be more precise, let $x(t)=x+v_{0} t, v(t)=v_{0}$ be the initial motion of a particle, and let $x(t)=x^{(i)}(x)+v^{(i)}(x) t, v(t)=v^{(i)}(x)$ be its motion between the $i$ th and the $(i+1)$ th reflections, $i=0,1, \ldots, m$. Let the body be invisible in the direction $v_{0}$; then one has $v^{(0)}=v^{(m)}=v_{0}, x^{(0)}=x$ and $x^{(m)}-x \perp v_{0}$. At each reflection and for any fixed $x$, the orientation of the triple $\left(\partial x^{(i)} / \partial x_{1}, \partial x^{(i)} / \partial x_{2}, v^{(i)}\right)$ changes. The initial and final orientations, $\left(\partial x^{(0)} / \partial x_{1}, \partial x^{(0)} / \partial x_{2}, v^{(0)}\right)$ and $\left(\partial x^{(m)} / \partial x_{1}, \partial x^{(m)} / \partial x_{2}, v^{(m)}\right)$, coincide, therefore $m$ is even.

On the other hand, $m$ cannot be equal to 2 , as seen from figure 7 . Therefore, $m \geqslant 4$.

From the examples of bodies discussed above, one concludes that the inequalities in (a) and (b) are sharp.

Finally, put some open questions.

(1) Do there exist bodies invisible in more than one direction? The same question concerns bodies of zero resistance/leaving no trace.

Note that bodies having zero resistance in a set of directions of positive Lebesgue measure do not exist. The proof will be published elsewhere.

(2) For which domains $\Omega$ (others than a ring) is theorem 2 true?

(3) The resistance of any convex body is non-zero. However, by taking a small portion of volume out of a convex body, one can get a body of zero resistance. Namely, there exists a sequence of zero resistance bodies $\mathcal{B}_{n}$ such that their relative volumes $\kappa\left(\mathcal{B}_{n}\right)$ go to 1 , $\lim _{n \rightarrow \infty} \kappa\left(\mathcal{B}_{n}\right)=1$. The maximal number of reflections for these bodies goes to infinity, $\lim _{n \rightarrow \infty} m\left(\mathcal{B}_{n}, v_{0}\right)=\infty$. The question is: estimate the maximal relative volume of a zero resistance body $\mathcal{B}$, given that the maximal number of reflections does not exceed a fixed value $m \geqslant 2$. In other words, estimate $\kappa_{m}:=\sup \left\{\kappa(\mathcal{B}): R_{v_{0}}(\mathcal{B})=0, m\left(\mathcal{B}, v_{0}\right) \leqslant m\right\}$. It is already known that $\kappa_{m} \geqslant 14 / 27$ and $\lim _{m \rightarrow \infty} \kappa_{m}=1$.

\section{Possible applications}

We believe that the models proposed in this paper can find application in optics and in aerodynamics of space flights.

A body of zero resistance with specular surface can be used, for example, as a constituent element of a structure (curtain) that lets light through only in one direction. By slightly modifying the construction, a surface can be designed that, like a lens, focuses sunlight onto one point. Bodies with mirror surface invisible in one direction may also be of interest. 
Above $150 \mathrm{~km}$, the atmosphere is so rarefied that the effect of intermolecular collisions is negligible [17]. As regards the body (union of two prisms) in figure 2(a), the flow density in some zones between the prisms duplicates and triplicates as compared with the density outside the body, that is, remains sufficiently small. The bodies depicted in figures $2(a)$ and 3 create infinite density along their symmetry axis; however this effect may be of little importance for practice, because of thermal motion of flow particles and not completely specular reflection from the body surface.

Our model is robust with respect to small changes in physical parameters. This means that in the case of slight thermal motion of gas molecules and nearly specular gas-surface interaction, the resistance is still small. The velocity of artificial satellites on low Earth orbits is much greater than the mean thermal motion of the atmospheric particles [18]. The gas-surface interaction is being intensively studied nowadays. It is very sensitive to many factors, including the spacecraft material, the atmosphere composition (which in turn depends on the height), the angle of incidence, and the velocity of the satellite. It is commonly accepted now that the interaction of the atmospheric particles with the surface of existing spacecraft at heights between 150 and $300 \mathrm{~km}$ is mostly diffuse [17, 19]; however it is argued [18] that carefully manufactured clean smooth metallic surfaces would favour specular reflections.

Therefore we believe that spacecraft of the shapes indicated in figures $2(a)$ and $(b)$ with suitably manufactured surfaces may experience reduced air resistance and, consequently, have increased lifetime and decreased deflection from the predicted trajectory.

\section{Acknowledgments}

This work was supported by the Centre for Research on Optimization and Control (CEOC) from the 'Fundação para a Ciência e a Tecnologia' (FCT), cofinanced by the European Community Fund FEDER/POCTI, and by FCT: research project PTDC/MAT/72840/2006.

\section{References}

[1] Newton I 1687 Philosophiae Naturalis Principia Mathematica (London: Streater)

[2] Legendre A M 1788 Memoires de L'Academie royale de Sciences annee 1786 (Paris: Chez Bachelier, Libraire) pp 7-37

[3] Belloni M and Kawohl B 1997 A paper of Legendre revisited Forum Math. 9 655-68

[4] Belloni M and Wagner A 2003 Newton's problem of minimal resistance in the class of bodies with prescribed volume J. Convex Anal. 10 491-500

[5] Buttazzo G and Kawohl B 1993 On Newton's problem of minimal resistance Math. Intell. 15 7-12

[6] Buttazzo G, Ferone V and Kawohl B 1995 Minimum problems over sets of concave functions and related questions Math. Nachr. 173 71-89

[7] Brock F, Ferone V and Kawohl B 1996 A symmetry problem in the calculus of variations Calc. Var. 4 593-9

[8] Buttazzo G and Guasoni P 1997 Shape optimization problems over classes of convex domains J. Convex Anal. $4343-51$

[9] Lachand-Robert T and Oudet E 2006 Minimizing within convex bodies using a convex hull method SIAM J. Optim. 16 368-79

[10] Lachand-Robert T and Peletier M A 2001 Newton's problem of the body of minimal resistance in the class of convex developable functions Math. Nachr. 226 153-76

[11] Comte M and Lachand-Robert T 2001 Newton's problem of the body of minimal resistance under a single-impact assumption Calc. Var. Partial Diff. Eqns 12 173-211

[12] Comte M and Lachand-Robert T 2001 Existence of minimizers for Newton's problem of the body of minimal resistance under a single-impact assumption J. Anal. Math. 83 313-35

[13] Plakhov A Yu 2003 Newton's problem of a body of minimal aerodynamic resistance Dokl. Akad. Nauk 390 314-7 
[14] Plakhov A Yu 2003 Newton's problem of the body of minimal resistance with a bounded number of collisions Russ. Math. Surv. 58 191-2

[15] Reed M and Simon B 1979 Methods of Modern Mathematical Physics, vol 3, Scattering Theory (New York: Academic Press)

[16] Petkov V 1980 High frequency asymptotics of the scattering amplitude for non-convex bodies Commun. Partial Differ. Eqns 5 293-329

[17] Harrison I K and Swinerd G G 1996 A free molecule aerodynamic investigation using multiple satellite analysis Planet. Space Sci. 44 171-80

[18] Fieseler P D 1998 A method for solar sailing in a low Earth orbit Acta Astronautica 43 531-41

[19] Moe K and Moe M M 2005 Gas-surface interactions and satellite drag coefficients Planet. Space Sci. 53 793-801 